\title{
Titanium-Carbide MXenes for Work Function and Interface Engineering in Perovskite Solar Cells
}

\author{
A. Agresti(a,b)§, A. Pazniak(c)§, S. Pescetelli(a)§, A. Di Vito(a), D. Rossi(a), A. Pecchia(d), M. Auf der \\ Maur(a), A. Liedl(e), R. Larciprete(e,f), Denis V. Kuznetsov(c), D. Saranin(b), A. Di Carlo(a,b)*
}

a) CHOSE - Centre for Hybrid and Organic Solar Energy, Department of Electronic Engineering, University of Rome Tor Vergata, via del Politecnico 1, 00133, Rome, Italy;

b) LASE - Laboratory of Advanced Solar Energy, National University of Science and Technology "MISiS", Leninsky prospect 4, 119049, Moscow, Russia.

c) Department of Functional Nanosystems and High-Temperature Materials National University of Science and Technology "MISiS", Leninsky prospect 4, 119049, Moscow, Russia

d) Istituto per lo Studio Materiali Nanostrutturati - CNR, Via Salaria km 29.600, 00014, Rome, Italy.

e) INFN-LNF, P.O. box 13, 00044 Frascati (Rome) Italy.

f) CNR-Institute for Complex Systems (ISC), Via dei Taurini 19, 00185 Rome, Italy

$\S:$ The authors contributed equally to this work

*corresponding author: aldo.dicarlo@uniroma2.it

\section{ABSTRACT}

In order to improve the efficiency of perovskite solar cells (PSCs), careful device design and tailored interface engineering are needed to enhance optoelectronic properties and the charge extraction process at the selective electrodes. Here, we use two-dimensional transition metal carbides (the MXene $\mathrm{Ti}_{3} \mathrm{C}_{2} \mathrm{~T}_{\mathrm{x}}$ ) with various termination groups $\left(T_{x}\right)$ to tune the work function (WF) of the perovskite absorber and the $\mathrm{TiO}_{2}$ electron transport layer (ETL), and to engineer the perovskite/ETL interface. Ultraviolet photoemission spectroscopy measurements and Density Functional Theory calculations show that the addition of $\mathrm{Ti}_{3} \mathrm{C}_{2} \mathrm{~T}_{\mathrm{x}}$ to halide perovskite and $\mathrm{TiO}_{2}$ layers permits to tune the materials' WFs, without affecting other electronic properties. Moreover, the dipole induced by the $\mathrm{Ti}_{3} \mathrm{C}_{2} \mathrm{~T}_{x}$ at the perovskite/ETL interface can be used to change the band alignment between these layers. The combined action of WF tuning and interface engineering can lead to substantial performance improvements in MXene-modified PSCs, as shown by the $26 \%$ increase of power conversion efficiency and hysteresis reduction with respect to reference cells without Mxene.

The rapid development of perovskite solar cells (PSCs) carried out in the last decade demonstrated the potentiality of this PV technology to compete on equal footing with traditional inorganic PV or to work in synergy with established silicon technology in tandem cell configuration. ${ }^{1}$ The remarkable efforts devoted to optimize the PSCs in term of device architecture ${ }^{2}$, perovskite composition ${ }^{3}$ and charge collecting electrodes ${ }^{4}$ produced a large wallet of emerging materials comprising organic polymers, ${ }^{5}$ oxides, ${ }^{6}$ two-dimensional (2D) materials $s^{7}$ and others ${ }^{8}$ able to tune the device properties ${ }^{9}$ and eventually to boost their performance and stability. ${ }^{10}$ Usually, a typical PSC is composed of a perovskite active layer, sandwiched between two selective charge transport layers (CTLS) and electrodes for negative and positive charge extraction. These are selected carefully by taking into account their compatibility with the underneath layers, ${ }^{11}$ their charge mobility ${ }^{12}$ and the energy level alignment with the perovskite absorber. ${ }^{13}$ However, being PSCs composed of several layers, interfaces ultimately play a crucial role in ruling device performance and stability. Charge transfer at the interfaces ${ }^{14}$ interface band alignment, ${ }^{15}$ interfacial vacancies, ${ }^{16}$ defects due to poor adhesion between layers ${ }^{17}$ and energy barriers have a strong impact on electrical device characteristics. From here, the pivotal role that interface engineering $(\mathrm{IE})^{18}$ has recently gained in the PSCs field. ${ }^{19}$ In this regard, 2D materials can be inserted as inter ${ }^{20}$ or buffer layers ${ }^{21}$ by modifying the chemical/physical properties of involved layers at 
the interface and eventually to improve the charge injection/collection at perovskite/CTLs interfaces. One of the main potentialities of 2D materials is the possibility to easily tailor their electronic structure, such as work function (WF $)^{22,23}$ or band gap, by proper functionalization ${ }^{24}$ or by quantum confinement. ${ }^{25}$ Fine-tuning of the WF allows to obtain appropriate energy level alignment leading to an ideal energy offset between perovskite active layer and CTLs, eventually inducing a built-in potential for efficient charge collection at the electrodes. Recently, a new family of 2D transition metal carbides, nitrides and carbonitrides (MXenes) ${ }^{26}$ with a general formula $M_{n+1} X_{n} T_{x}(n=1,2,3)$, where $M$ represents an early transition metal, $X$ is a carbon and/or nitrogen atom and $T_{X}$ the surface-terminating functional groups came out as a promising class of 2D materials in many applications $^{26,27}$ owing to their physical and chemical properties. ${ }^{26,28-30}$ Rich chemistry and surface termination make MXenes unique 2D materials with huge possibilities to tune their electronic properties. In fact, MXenes offer the possibility to vary the WF by choosing the proper transition metal as well as the $X$ element. ${ }^{31}$ Moreover, during the synthesis of MXenes, their surfaces are naturally functionalized, which changes the electrostatic potential near the surfaces and affects the electronic structure, significantly shifting the WF. ${ }^{32}$ As density functional theory (DFT) predicts, surface termination strongly influences the density of states ${ }^{33}$ and the WF of MXenes ${ }^{34}$ which can range from $1.6 \mathrm{eV}$ (for OH-termination) to $6.25 \mathrm{eV}$ (for Otermination). ${ }^{34,35}$ This opens new opportunities for MXenes applications in optoelectronics and in particular in photovoltaics where already some initial studies have been presented for organic solar cells ${ }^{36}$, Si solar cells, ${ }^{37}$ dye-synthesized solar cells ${ }^{38}$ and PSCs. ${ }^{39,}{ }^{40}$ In the PSC case, $\mathrm{Ti}_{3} \mathrm{C}_{2} \mathrm{~T}_{\mathrm{x}}$ MXenes have been incorporated into the perovskite absorber showing an improved morphology and an enhanced PCE $(+12 \%)$ with respect to the reference cell without $\mathrm{MXenes}{ }^{39}$ or into the $\mathrm{SnO}_{2}$ electron transporting layer $(\mathrm{ETL})^{40}$ to provide superior charge transfer paths that permits to enhance the PCE $(+6.5 \%)$ with respect to the reference cells.

In order to reveal the role of MXenes in perovskites and provide a clear strategy on the use of MXenes for WF and IE in PSCs, we synthesized $\mathrm{Ti}_{3} \mathrm{C}_{2} \mathrm{~T}_{\mathrm{x}}$ MXene and performed an extensive characterization of the MXene structure and electronic properties (Figure 1).
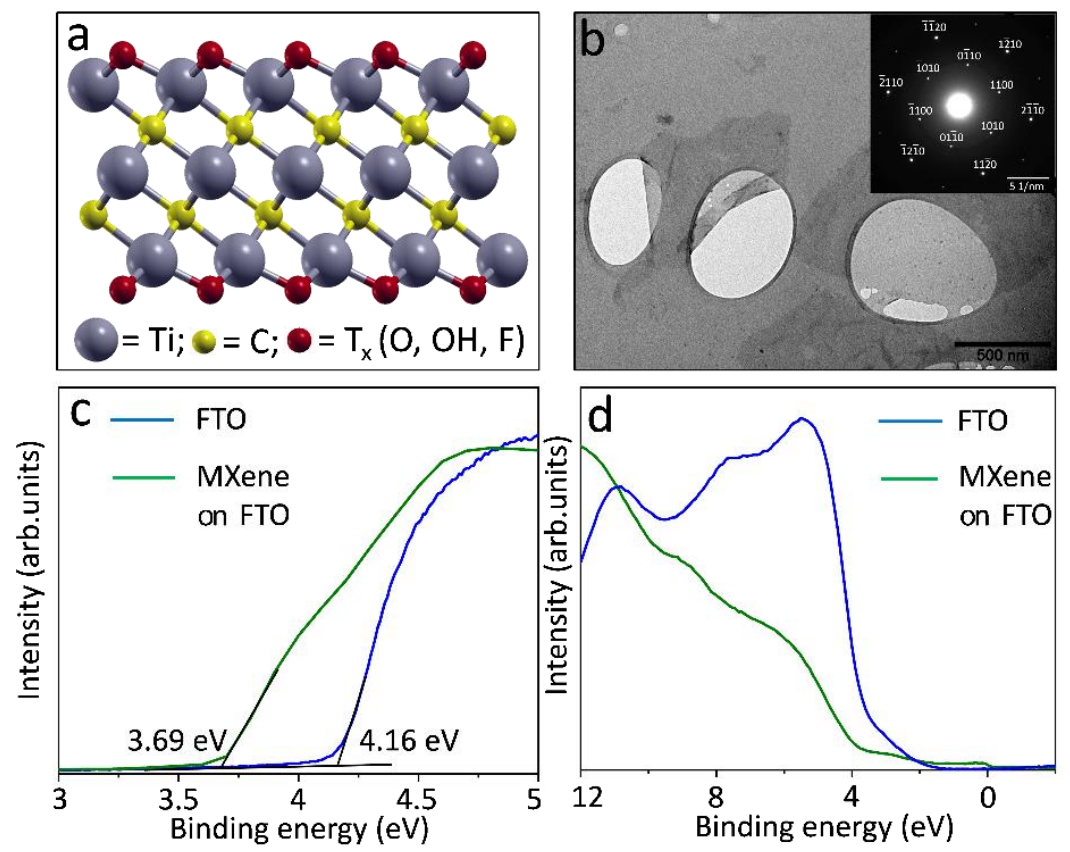

Figure 1: Characterization of $\mathrm{Ti}_{3} \mathrm{C}_{2} \mathrm{~T}_{x}$ MXene. a, Schematic structure of $\mathrm{Ti}_{3} \mathrm{C}_{2} \mathrm{~T}_{x} M$ Mene. Surface terminations $\left(T_{x}\right)$ are a mixture of $\mathrm{F}, \mathrm{O}$, and $\mathrm{OH}$. b, TEM image of $\mathrm{Ti}_{3} \mathrm{C}_{2} \mathrm{~T}_{x} \mathrm{MXene}$ flakes. The corresponding selected area electron diffraction (SAED) pattern is reported in the inset. UPS spectra measured with photon energy of $40.81 \mathrm{eV}$ on the MXene flakes and FTO substrate supporting them are reported in left panel $\mathbf{c}$ and right panel $\mathbf{d}$ showing secondary electron cut-off and valence band region, respectively. 
After chemical etching and exfoliation by sonication, single-layer $\mathrm{Ti}_{3} \mathrm{C}_{2} \mathrm{~T}_{x}$ nanosheet (Figure 1a) consists of two carbon atoms which bind three titanium ones as elementary units. The outer Ti layers tend to terminate with functional groups $(\mathrm{Tx})$ such as $\mathrm{O}, \mathrm{OH}$, and $\mathrm{F}$ (Figure 1a), which are randomly distributed on the MXene surface. The MXene structure is characterized by the presence of single atomically-thin transparent flakes of 2D titanium carbides with hexagonal symmetry which follows the parent MAX phase after exfoliation (Figure 1b). $\mathrm{Ti}_{3} \mathrm{C}_{2} \mathrm{~T}_{\mathrm{x}}$ sheets have irregular edges and size distribution ranging generally from 1.5 to $2.5 \mu \mathrm{m}$. Details about MXene composition were extracted by XPS measurements reported in Figures S1 and S2a. In particular, the ratio between the $\mathrm{F}: \mathrm{OH}: \mathrm{O}$ functional groups is estimated to be $1.6: 0.65: 0.34$. Based on these results, it can be concluded that the MXene surface randomly ends with the $\mathrm{F}, \mathrm{OH}$ and $\mathrm{O}$ groups, with a prevalence of fluorine functional groups.

The electronic properties of $\mathrm{Ti}_{3} \mathrm{C}_{2} \mathrm{~T}_{\mathrm{x}}$ was featured by WF measurement using ultraviolet photoelectron spectroscopy (UPS). We found that the WF of synthesized MXene flakes deposited on FTO glass is rather low and equal to $3.7 \mathrm{eV}$ (Figure 1c). The spectra measured in the valence band region (Figure 1d) show that the intensity extends up to the Fermi level and severely increases for binding energy (BE) close to $4 \mathrm{eV}$, likely due to the contribution of the oxidized phases detected in the XPS spectra (see Figure S1a,b). In general, the reported UPS measured WF values for $\mathrm{Ti}_{3} \mathrm{C}_{2} \mathrm{~T}_{\mathrm{x}} \mathrm{MXene} \mathrm{range} \mathrm{from} 3.4 \mathrm{eV}^{41}$ to $4.62 \mathrm{eV}{ }^{42}$ Such large modulation of the WF could be attributed to the different etching environment dramatically affecting the surface termination. The WF value of synthesized MXene is in agreement with the XPS results and should be due to the substantial percentage of hydroxyl groups terminating the $\mathrm{Ti}_{3} \mathrm{C}_{2} \mathrm{~T}_{\mathrm{x}}$ matrix. This finding is supported by theoretical calculations ${ }^{32}$ (see also the discussion in $\mathrm{SI}$ ) and experimental observations reported in literature. ${ }^{41}$

The low WF of MXene flakes could be exploited to finely control the energy level alignment between the perovskite absorber layer and the CTLs. To this end, we probed the effect of the MXene employed as additive in perovskite and $\mathrm{TiO}_{2}$ layers onto their WFs by combining UPS (Figure 2) and XPS (Figure S2b) measurements. ${ }^{43}$ The WF of pristine perovskite, determined from the secondary electron onset, is $4.72 \mathrm{eV}$, which is shifted to $4.37 \mathrm{eV}$ after the addition of MXene (see Figure 2a), i.e. $0.35 \mathrm{eV}$ lower than that of the undoped perovskite. The valence band spectrum measured on the MXene-doped perovskite film and the energy gap appear quite like the pristine ones. (Figure $\mathbf{2 b}$ and $\mathbf{S 3}$ ). The energy diagram in Figure $\mathbf{2 c}$ schematizes the WF and Ionization Energy (IE) reduction of MXene-doped perovskite.
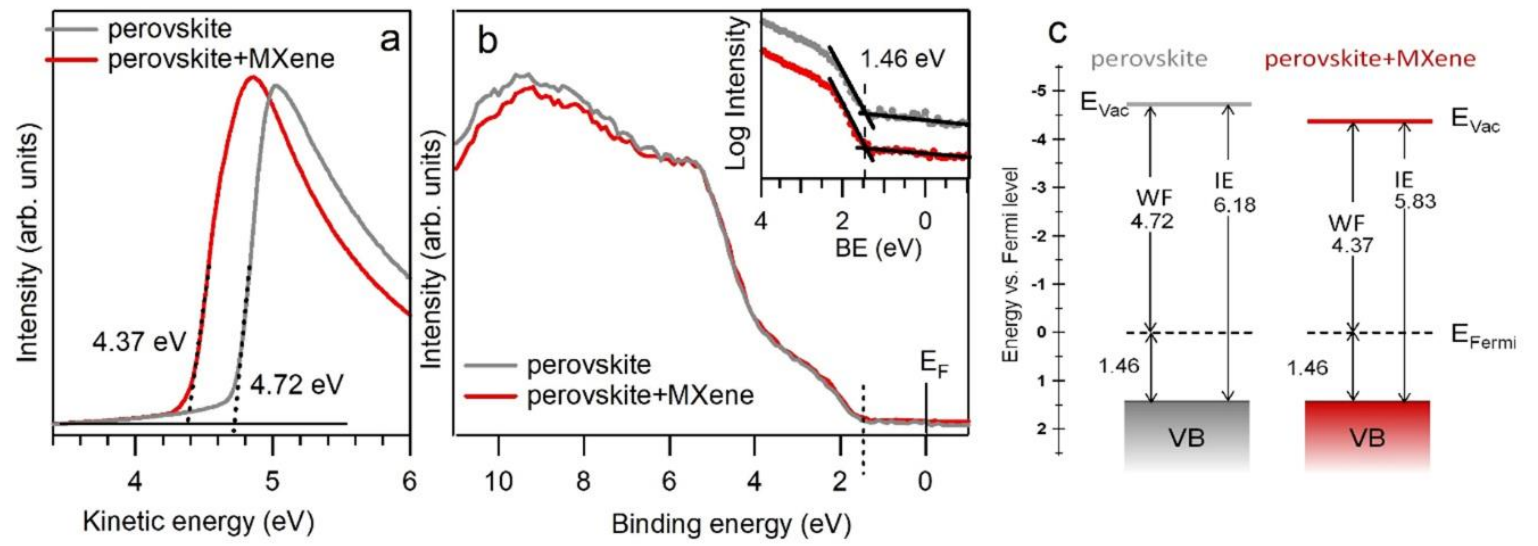

Figure 2: UPS curves of pristine and MXene-doped perovskite films. a, UPS spectra around the secondary electron cut-off. b, UPS spectra in the valence band region. For the pristine perovskite, the valence band maximum (VBM), determined by the intercept to zero of the intensity plotted in logarithmic scale (see inset in panel $\mathbf{b}$ ) is at $1.46 \mathrm{eV}$ below the Fermi level, in good agreement with previous findings. ${ }^{43}$ c, Energy scheme for undoped and MXene-doped perovskite with respect to the Fermi level. 
We exclude that in perovskite deposition the addition of MXene can change the perovskite crystal growth and the resulting morphology since no significant changes have been observed in the scanning electron microscopy (SEM) images reported in SI (Figure S4).

UPS characterizations were performed also for the $\mathrm{TiO}_{2}$ ETL with and without addition of MXene (Figure S5). In this case, MXene doping leaves the shape and the maximum of the valence band almost unchanged. At the same time, the WF slightly decreases from $3.91 \mathrm{eV}$ for the $\mathrm{TiO}_{2}$ sample to $3.85 \mathrm{eV}$ in the case of MXenedoped $\mathrm{TiO}_{2}$. The small WF differences between the pristine and doped $\mathrm{TiO}_{2}$ layers compared to the WF shift induced by MXene in perovskite can be related to the oxidation of $\mathrm{Ti}_{3} \mathrm{C}_{2} \mathrm{~T}_{\mathrm{x}}$ MXene (Figure S6). However, the WF decrease observed in the doped $\mathrm{TiO}_{2}$ layer suggests that $\mathrm{MXene}$ does not oxidize completely when annealed with $\mathrm{TiO}_{2}$ as confirmed by the XRD pattern measured for sintered MXene-doped $\mathrm{TiO}_{2}$ (Figure S7). In order to understand the origin of the perovskite WF change induced by the interaction with MXene, we performed DFT calculations of the perovskite/ $\mathrm{Ti}_{3} \mathrm{C}_{2} \mathrm{~T}_{\mathrm{X}}$ with $\mathrm{OH}$ and $\mathrm{O}$ termination. To make this calculation feasible, we consider the single cation $\mathrm{CH}_{3} \mathrm{NH}_{3} \mathrm{Pbl}_{3}\left(\mathrm{MAPbl}_{3}\right)$ perovskite having experimentally verified that MXene doping induces a WF shift also for this perovskite (Figure S8). Calculation details are reported in SI (see Figures S9 and S10). DFT calculations reported in Figure 3 show that the charge transfer at the $\mathrm{MAPbl}_{3} / \mathrm{Ti}_{3} \mathrm{C}_{2}(\mathrm{OH})_{2}$ interface induces the formation of an interface dipole, causing an important reduction of the WF and affecting the band alignment of the system. The transferred charge density is localized at the very first $\mathrm{Pb}-\mathrm{l}$ layer, therefore involving less than $1 \mathrm{~nm}$ of the surface. The formation of an interface dipole can also be deduced from the vacuum potential slopes observed in Figure $\mathbf{3 a}$ and $\mathbf{3 b}$. In the case of $\mathrm{OH}$ terminated $\mathrm{MXene}$, the slope is higher than $\mathrm{O}$ termination, implying a larger interface dipole. Moreover, in $\mathrm{MAPbl}_{3} / \mathrm{Ti}_{3} \mathrm{C}_{2} \mathrm{O}_{2}$ the potential slope (i.e. the interface dipole) has the opposite direction with respect to $\mathrm{OH}$ termination case. Figure $\mathbf{3} \mathbf{c}$ and $\mathbf{3} \mathbf{d}$ show the projected bands of the $\mathrm{MAPbl}_{3} / \mathrm{MXene}$ slabs, where the red colour represents the contribution from the inner atoms of the $\mathrm{MAPbl}_{3}$ slab, falling in the grey boxes drawn in Figure $\mathbf{3 a}$ and $\mathbf{3 b}$. 

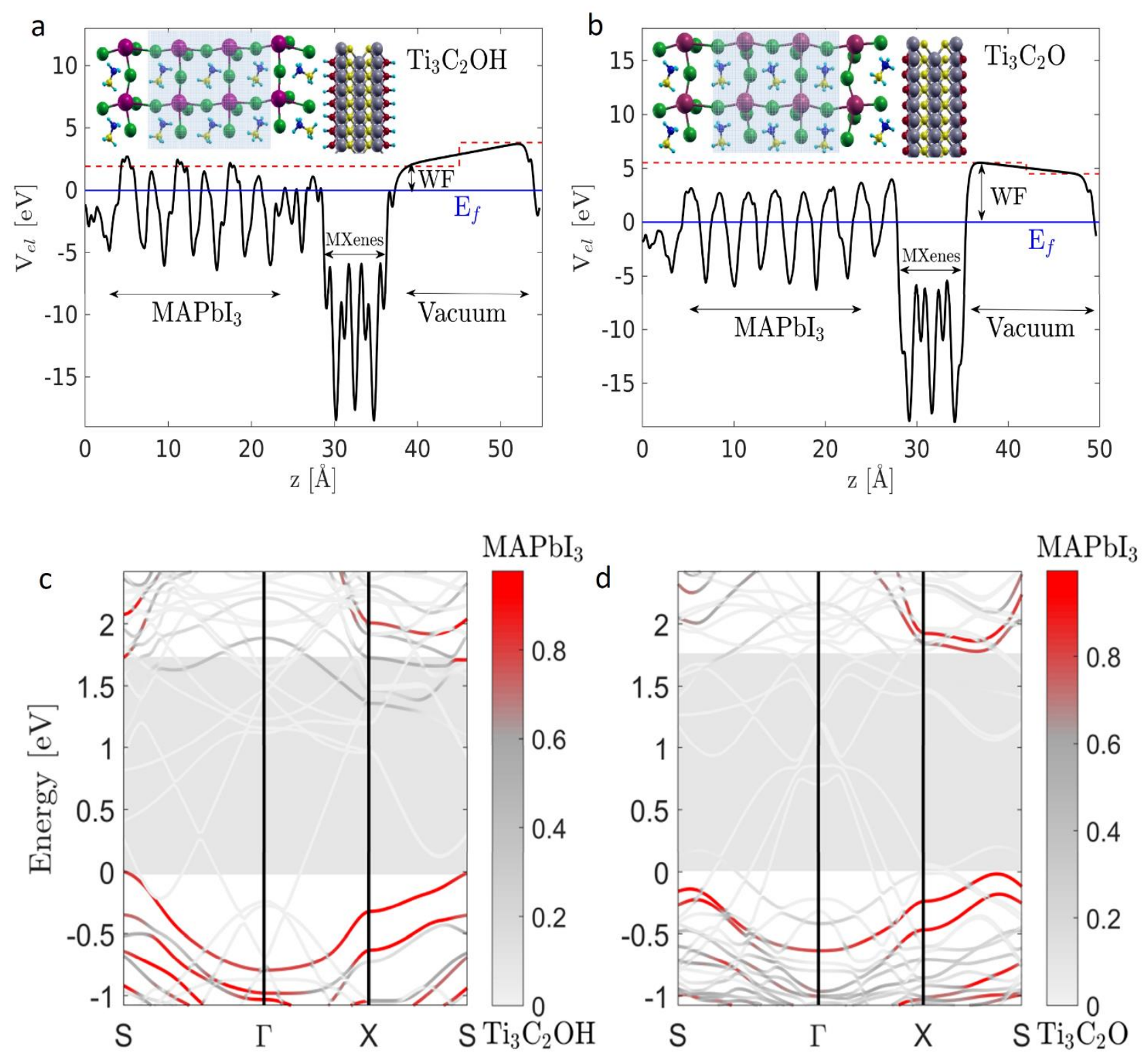

Figure 3: DFT calculation of the $\mathrm{MAPbl}_{3} / \mathrm{MXene}$ structure. a, b Electrostatic potential averaged over planes perpendicular to the $\mathrm{MAPbl}_{3} / \mathrm{Ti}_{3} \mathrm{C}_{2}(\mathrm{OH})_{2}$ and $\mathrm{MAPbl}_{3} / \mathrm{Ti}_{3} \mathrm{C}_{2} \mathrm{O}_{2}$ interface, respectively. The computed structures are shown within the plots, where green, magenta, blue, yellow, cyan, grey and red spheres represent $\mathrm{I}, \mathrm{Pb}, \mathrm{N}, \mathrm{C}, \mathrm{H}, \mathrm{Ti}$ and $\mathrm{O}$ atoms, respectively. The red dashed lines represent the dipole corrected vacuum levels. The Fermi energy is set to zero, so that the vacuum potential, just away from the MXene surface, corresponds to the WF, of the $M A P b{ }_{3} / T_{3} C_{2} T_{x}$ interface, as depicted in the panels. We can see that the WF derived for the $\mathrm{OH}$ terminated MXene configuration,

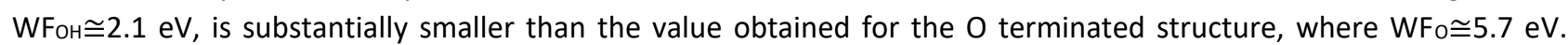
Notably, similar calculations for the $\mathrm{F}$ terminated MXene do not show a significant variation of $\mathrm{MAPbl}_{3} \mathrm{WF}$. c, d, Projected band structures of the $\mathrm{MAPbl}_{3} / \mathrm{MXene}$ slabs for $\mathrm{OH}$ and $\mathrm{O}$ termination of the $\mathrm{MXene}$, respectively. Contribution from the bulk part of the $\mathrm{MAPbl}_{3}$ slab (grey box in panels $\mathbf{a}, \mathbf{b}$ ) are coloured in red. The valence band edge is set to zero. The bulk band gap of the $\mathrm{MAPbl}_{3}$ is indicated by the shaded area.

A remarkable outcome emphasised in Figures $\mathbf{3 c}$ and $\mathbf{3 d}$ is that, even in such small structures, the $\mathrm{MAPbl}_{3}$ bandgap is barely affected by the interaction with MXene, remaining at $1.7 \mathrm{eV}$. This gap independence is a feature of MXene additives also seen in experiments. We conclude that the interaction is mainly electrostatic, owing to the dipole formation at the interface. A similar result is obtained when considering a symmetric 
structure MXene/MAPbl 3 /MXene (see Figure S11), demonstrating that the WF shift of perovskite also occurs when MXene is found at both ETL and HTL interfaces or within the bulk of the material, decreasing its averaged WF. In our calculations we have assumed that different cations (MA- methylammonium, FAformamidinium, Cs- cesium) behave similarly, although they affect the lattice constants and the bandgap. For instance, in the case of FA, shown in Figure $\mathbf{S 1 2}$, the WF reduces from $4.5 \mathrm{eV}$ of bare perovskite to $1.7 \mathrm{eV}$, in contrast to $2.1 \mathrm{eV}$ obtained for MA. On the contrary, we expect that the WF of $\mathrm{CsPbl}_{3}+\mathrm{Ti}_{3} \mathrm{C}_{2} \mathrm{OH}$ will be larger, owing to a larger bandgap that results in a smaller interfacial polarization. Our multication perovskite have nominal fractions $\mathrm{FA}=78.85 \%, \mathrm{MA}=16.15 \%, \mathrm{Cs}=5 \%$, hence $\mathrm{Cs}$ plays a minor role.

We also observe that the WF reduction induced by the $\mathrm{OH}$ terminated surface is much more pronounced than the WF increase induced by $O$ terminations. This is due to a rather strong non-linear behaviour already observed in literature ${ }^{35}$ and also obtained in our calculations. Indeed, the perovskite WF using MXene with a $\mathrm{OH}: \mathrm{O}$ termination ratio of 50:50 reduces to $3.1 \mathrm{eV}$, whereas for a ratio of 75:25 it is close to $1.9 \mathrm{eV}$, namely close to the value obtained for $100 \% \mathrm{OH}$. Based on the XPS data reported above (Figure S1,S2), which estimate $62 \% \mathrm{~F}, 25 \% \mathrm{OH}$ and $13 \% \mathrm{O}$ terminations, whereas the plain $\mathrm{OH}: \mathrm{O}$ ratio would determine a $\Delta \mathrm{WF} 2$ we expect the reduction of WF to be 4 times smaller due to the abundance of fluorine, hence $\Delta \mathrm{WF} \cong-0.5 \mathrm{eV}$, much closer the value of $-0.35 \mathrm{eV}$ experimentally observed. Similar calculations reported in $\mathrm{SI}$ show the impact of MXene on the WF of $\mathrm{TiO}_{2}$ (see Figure S13). Even in this case, the WF obtained for the anatase (101) surface with $\mathrm{OH}$ terminated $\mathrm{MXene}, \mathrm{WF}_{\mathrm{OH}} \cong 1.6 \mathrm{eV}$, is smaller than that with $\mathrm{O}$ terminated $\mathrm{MXene}$, where $W F_{0} \cong 5.8 \mathrm{eV}$.

Five different PSC structures were designed and characterized to investigate the impact of $\mathrm{Ti}_{3} \mathrm{C}_{2} \mathrm{~T}_{\mathrm{x}} \mathrm{MXene}$. In particular, the "reference" device has a typical layer sequence with compact $\left.(\mathrm{cTIO})_{2}\right)$ and mesoporous $\left(\mathrm{mTiO}_{2}\right)$ $\mathrm{TiO}_{2}$, namely glass/FTO/cTiO $/ \mathrm{mTiO}_{2} /$ perovskite/spiro-OMeTAD/Au, while the other structures include MXene as a dopant or as interlayer as summarized in Table 1. The comparison of cross-section SEM images for reference and full-MXene device (type C), reported in Figure S14, exclude a direct impact of MXene on the device layer morphology.

Name

Reference

Type A

Type B

Type C Type D*
Device Structure

Glass/FTO/cTiO $/ \mathrm{mTiO}_{2} /$ perovskite/spiro-OMeTAD/Au

Glass/FTO/cTiO $/ \mathrm{mTiO}_{2} /$ perovskite+MXene/spiro-OMeTAD/Au

Glass/FTO/cTiO ${ }_{2}+\mathrm{MXene} / \mathrm{mTiO}_{2}+\mathrm{MXene} /$ perovskite+MXene/spiro-OMeTAD/Au

Glass/FTO/cTiO + + MXene/mTiO ${ }_{2}+\mathrm{MXene} / \mathrm{MXene} /$ perovskite+MXene/spiro-OMeTAD/Au Glass/FTO/cTiO $2 / \mathrm{mTiO}_{2}+\mathrm{MXene} / \mathrm{MXene} /$ perovskite+MXene/spiro-OMeTAD/Au

Table 1: Structure of the investigated PSCs. All device types (A, B, C and D) are based on MXene-doped perovskite. Type $\mathrm{B}$ and $\mathrm{C}$ devices include also $\mathrm{MXene}$ doping in $\mathrm{CTiO}_{2}$ and $\mathrm{mTiO}_{2}$. The structure $\mathrm{C}$ has an additional MXene interlayer at the $\mathrm{mTiO}_{2}$ /perovskite interface. Devices of type $\mathrm{D}$, similar to $\mathrm{C}$ but with a standard $\mathrm{CTiO}_{2}$ layer, have been fabricated to identify the role of MXene in the $\mathrm{CTiO}_{2}$ layer. *Discussions related to structure D are reported in SI.

As a main result, all device structures employing MXene (type A, B, C Figure 4 and D Figure S15) show an average improved PCE with respect to reference cells ( $+26.5 \%$ for type $C$ ) mainly due to an average improvement in $\mathrm{V}_{\mathrm{OC}}(+5.7 \%)$ and $\mathrm{FF}(+13.7)$ with respect to the reference device. A less marked, but still important improvement in averaged $\mathrm{J}_{\mathrm{sc}}$ was also observed ( $+4.8 \%$ for type $\mathrm{C}$ ).

A remarkable efficiency of $20.14 \%$ is achieved in the case of full-MXene structure (Type C) with $F F=77.6 \%$, $\mathrm{J}_{\mathrm{SC}}=23.82 \mathrm{~mA} / \mathrm{cm}^{2}$ and $\mathrm{V}_{\mathrm{OC}}=1.09 \mathrm{~V}$. Notably Type C PSCs exhibit a very good stabilized power under prolonged 1 SUN irradiation at maximum power point (MPP), as reported in Figure S16a. The incident photon to current conversion efficiency (IPCE) spectrum for Type $C$ devices is reported in Figure $\mathbf{S 1 6}$, together with the integrated short circuit current, while the related J-V characteristics are reported in Figure S17a. As shown by the J-V characteristics, a clear benefit in the use of MXene in PSC consists in a significant reduction of the J-V hysteresis, particularly relevant in the case of type $\mathrm{C}$. The hysteresis index $(\mathrm{HI})$, calculated as the ratio 
between the PCE extracted form reverse J-V scan (open circuit-> short circuit) and the one from the forward scan (short circuit-> open circuit), reduces in average from 1.22 of the reference cell to 1.04 of the Type $C$ MXene-based PSC (see Figure S17b). In addition, type $C$ and reference devices were exposed to continuous 1 SUN irradiation for 30 minutes. While the type $C$ device showed stabilized power output at MPP ( $\left.P_{\text {MPP }}\right)$ after 25 minutes by retaining more than $83 \%$ of initial value (see Figure S17c), the reference cell demonstrated a monotonic $P_{M P P}$ decrease trend after 10 minutes. The stabilization effect due to the MXene can be related to the improved charge extraction, since trapped charge at the interfaces are well-known to trigger the degradation. ${ }^{44}$
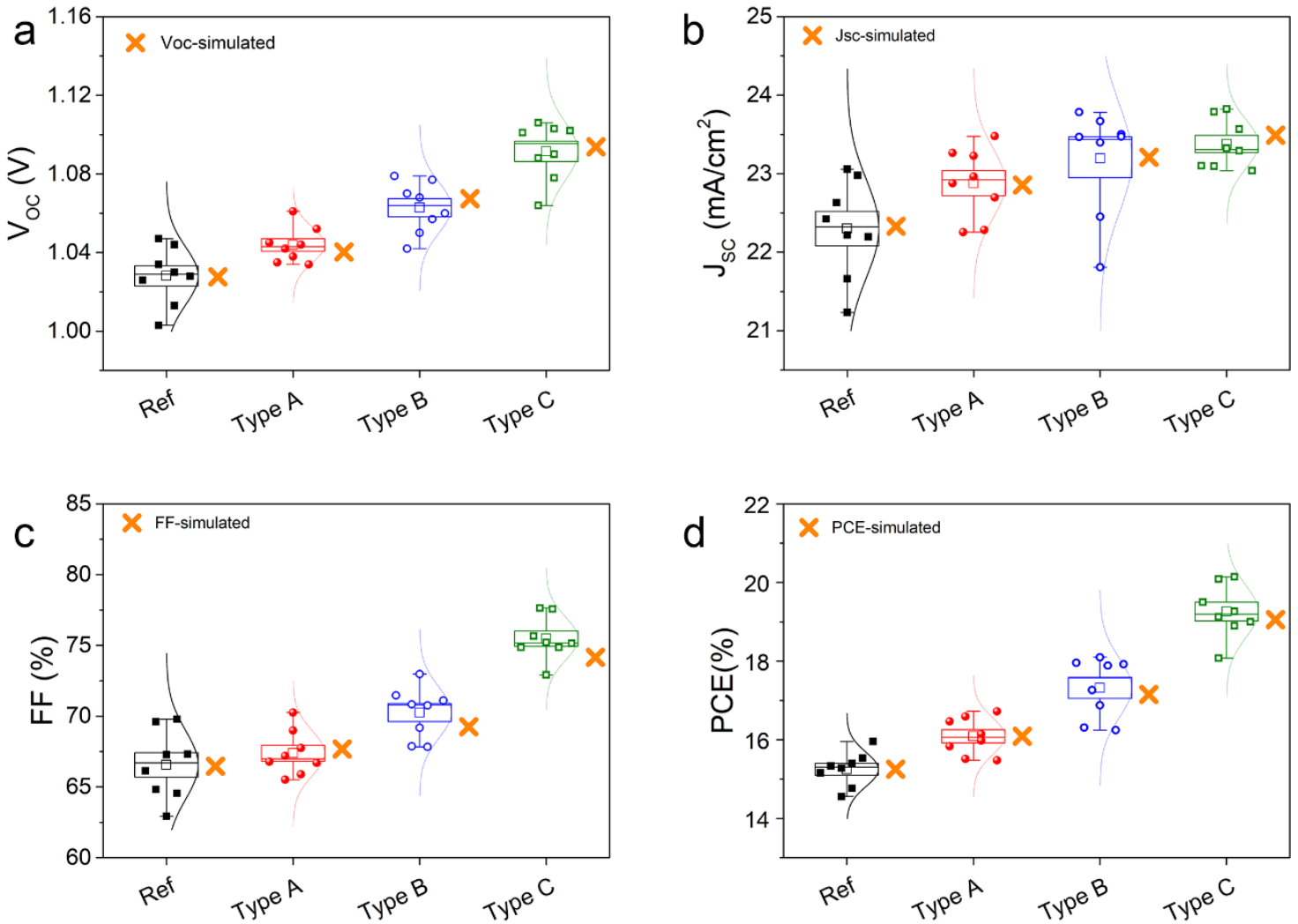

Figure 4: Photovoltaic parameter statistics for the investigated PSCs. a, Open circuit voltage (Voc). b, Short circuit current density (Jsc). c, Fill factor (FF). d, Power conversion efficiency (PCE). Parameters are extracted from the J-V curves acquired at 1 SUN irradiation. The standard error (SE) is represented with a box while the average value is depicted as an empty squared dot. For each PSC structure, 8 devices have been fabricated with a cell active area of $0.09 \mathrm{~cm}^{2}$. Results for device simulations are also displayed as orange crosses.

To further elucidate the role of MXene in each PSC structure, we performed transient photocurrent (TPC) and photovoltage (TPV) measurements combined with light power dependency of $J_{S C}$ and $V_{O C}$, photoluminescence spectra, charge carrier lifetime and charge extraction measurements (Figure S18). The insertion of MXene within both $\mathrm{mTiO}_{2}$ and perovskite layer reduces charge recombination rate at the $\mathrm{TiO}_{2}$ /perovskite interface (see SI and Figures S18a, S18b, S18c and S18d for details) while the MXene interlayer is able to reduce trap state density and/or passivate the $\mathrm{mTiO}_{2}$ surface (see $\mathrm{SI}$ and Figures S18e, S18f). Finally, when MXene is added to $\mathrm{CTiO}_{2}$ layer, energy barrier at the $\mathrm{CTiO}_{2} / \mathrm{mTiO}_{2}+\mathrm{MXene}$ interface is significantly reduced, by improving the electron collection at the photoelectrode (Figure S19).

In order to support the conclusion that the improvement of PSC performance are directly related to WF tuning, optimized interface alignment and reduction of charge recombination induced by MXene, we performed device simulations based on a drift-diffusion model ${ }^{45}$.

According to the experimental devices, we model the reference solar cell as a $\mathrm{FTO} / \mathrm{cTiO}_{2} / \mathrm{mTiO}_{2}$ +perovskite/Spiro-OMeTAD/Au stack, where the $\mathrm{mTiO}_{2}$ scaffold and perovskite are 
modelled as an effective $550 \mathrm{~nm}$ absorbing perovskite layer with band edges at $E_{\mathrm{C}}=-3.9 \mathrm{eV}$ and $\mathrm{E}_{\mathrm{V}}=-5.5 \mathrm{eV}$. ${ }^{46}$ Cell types A, B and C have been modelled by shifting the WFs of the MXene-doped layers according to the UPS measurements presented in Figure 2 and Figure S5. In particular, we applied a rigid upshift of the bandedges of perovskite (type A) and perovskite and $\mathrm{CTiO}_{2}$ layers (types $\mathrm{B}$ and $\mathrm{C}$ ) of $0.35 \mathrm{eV}$ and $0.05 \mathrm{eV}$, respectively. For device type $C$ we assumed that the MXene interlayer, owing to its low WF, further improves the band alignment at $\mathrm{TiO}_{2}$ /perovskite interface. This has been modelled by an additional upwards shift of the band edge of $\mathrm{CTiO}_{2}$. Additionally, we assumed a slightly smaller interface recombination at the $\mathrm{TiO}_{2}$ /perovskite interface for type $\mathrm{C}$ compared to type $\mathrm{B}$, based on the measured lifetimes and on the light intensity dependence of the $\mathrm{V}_{\mathrm{OC}}$ (see Figure $\mathbf{S 1 8 c}$, Figure $\mathbf{S 1 8 d}$ and discussion in SI). The full list of parameters employed in the simulations is reported in Table S1, while energy band profiles for the simulated device structures are reported in Figure S20. By comparing J-V simulations with experimental results (Figure S21) and simulated PV parameters with experimental ones (Figure and Figure S22), we can deduce that the proposed model is able to consistently reproduce experimental results for all PSC types.
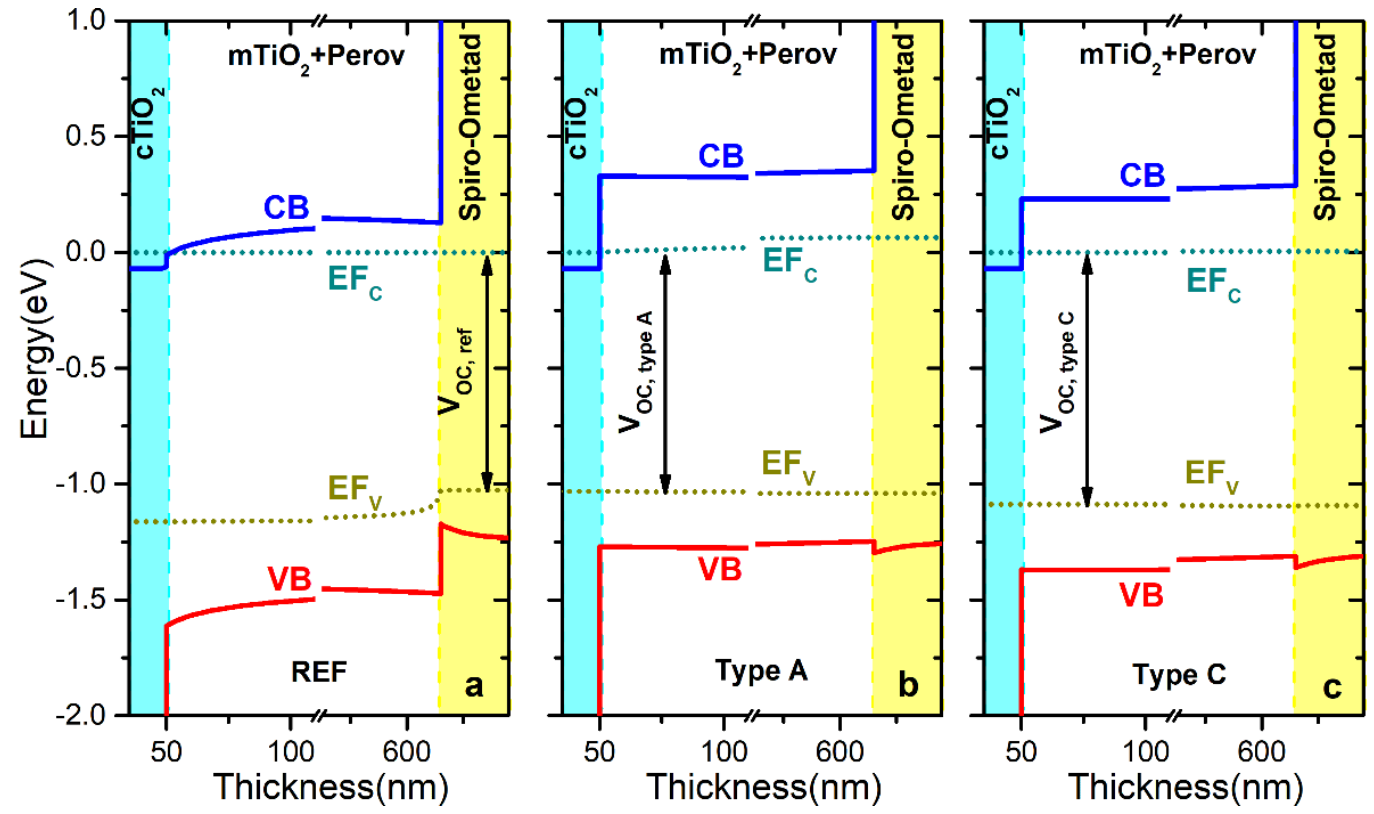

Figure 5: Band profiles of PSCs with and without MXene as obtained by physical simulation modelling. Panels show the Conduction Band (CB), Valence Band (VB) profiles at $V_{O c}$ between corresponding quasi Fermi levels for $C B$ ( $E F C$ ) and VB (EFV). a, Reference PSC. b, Type A PSC. c, Type C PSC.

Figure 5 shows the band profiles and quasi Fermi levels for the reference (Figure 5a), Type A (Figure $\mathbf{5 b}$ ) and Type C (Figure 5c) PSCs at open circuit conditions. The barrier at the perovskite/spiro-OMeTAD interface found in the valence band of the reference device, which is responsible for the $V_{o c}$ drop, reduces in MXenedoped perovskite for both type A and Type C PSCs. The reduced barrier at the perovskite/spiro-OMeTAD interface tends to increase the $\mathrm{V}_{\mathrm{oc}}$, however in Type A PSC the up-shift of the perovskite bands increase the band discontinuity at the $\mathrm{TiO}_{2}$ /perovskite interface with a consequent penalization of the final $\mathrm{V}_{\text {oc. }}$. This problem is partially solved in Type $\mathrm{C}$ (Figure $\mathbf{5 c}$ ) where the additional up-shift of the $\mathrm{TiO}_{2}$ band induced by the MXene doping and MXene interlayer reduces the $\mathrm{TiO}_{2}$ /perovskite barrier and improves the $\mathrm{V}_{\mathrm{oc}}$.

We should point out that the simulations with band shifting induced by the $\mathrm{Ti}_{3} \mathrm{C}_{2} \mathrm{~T}_{x}$ MXene cannot justify the increase of the $\mathrm{J}_{\mathrm{sc}}$ observed experimentally (see Figure $\mathbf{4 b}$ and Figure S22). However, MXene-doped perovskite showed and increased light absorption and IPCE with respect to pristine perovskite (see Figure S23). We can speculate that this could be related to scattering ${ }^{47}$ or plasmonic resonance ${ }^{48}$ induced by MXene into the perovskites even though a clear picture for this phenomenon has not been identified. Accounting for this absorption coefficient increase in the simulations, we are able to recover the increase of $J_{\text {sc }}$ (Figures S21 and S22). The increase of FF for Type C PSCs suggests a change of the transport properties at the 
$\mathrm{TiO}_{2}$ /perovskite interface induced by the MXene interlayer (see Figure $\mathbf{S 1 8}$ and discussion in $\mathrm{SI}$ ). By modelling this effect with an increase of carrier mobility in the effective $\mathrm{mTiO}_{2}$ /perovskite layer we are able finally to recover also the increase of FF (Figure S22), as demonstrated by comparing J-V simulations with experimental results (Figures S21 and S22).

\section{OUTLOOK}

This work focuses on the demonstration that MXenes, in particular the $\mathrm{Ti}_{3} \mathrm{C}_{2} \mathrm{~T}_{\mathrm{x}}$, owing to their WF tunability controlled by the surface termination groups can be exploited to modify the WF of perovskite and transporting layers with a consequent optimization of band alignments in PSCs. The WF shift has been observed for both single and multi-cation perovskite doped with $\mathrm{Ti}_{3} \mathrm{C}_{2} \mathrm{~T}_{x} \mathrm{MXene}$ by demonstrating the general applicability of our approach. We also show that MXene can be effectively employed to tune the interface between perovskite absorber and $\mathrm{TiO}_{2} \mathrm{ETL}$ to further enhance charge transfer between the two layers. By combining MXene-doped layers and MXene-engineered interfaces we are able to demonstrate a strong improvement of PSC efficiency (+26\%) with respect to the reference cell, achieving a final maximum efficiency exceeding $20 \%$ and an almost complete suppression of the cell hysteresis. The possibility to vary on demand the WF of materials and tuning their band alignments with other layers forming an electronic device is of fundamental importance to enlarge the design parameter space and to improve device performance. MXenes have the potentialities to be a key player in WF tuning of perovskite absorbers (Figure S24) and CTLS in PSCs, without affecting other properties of the materials. Owing to this unique property, we believe the MXene WF tuning and MXene interface engineering developed in this work can inspire innovative efficient designs of PSCs and other perovskite based devices such as LED and detectors.

\section{AUTHORS CONTRIBUTIONS}

An.P, ADC, DS, and DVK conceived the work. AA and SP performed the experiments on solar cells and the electro-optical characterizations. An.P produced and characterized the MXenes. ADV, DR, AI.P and MA performed the theoretical simulations. RL and AL performed UPS and XPS analysis. ADC coordinated the research activity. The manuscript was written through the contributions of all the authors. All the authors have given approval to the manuscript final version.

\section{ACKNOWLEDGEMENTS}

$A D C$ and DS gratefully acknowledge the financial support from the Ministry of Education and Science of the Russian Federation in the framework of MegaGrant № 075-15-2019-872 (14.Y26.31.0027/074-02-2018-327). AA and SP gratefully acknowledge funding from the European Union's Horizon 2020 Research and Innovation Program under grant agreement no.785219-GrapheneCore2.

\section{REFERENCES}

1. Cai, M. et al. Cost-Performance Analysis of Perovskite Solar Modules. Adv. Sci. 4, 1600269 (2016).

2. Hussain, I. et al. Functional materials, device architecture, and flexibility of perovskite solar cell. Emergent Mater. 1, 133-154 (2018).

3. Kim, H.-S., Hagfeldt, A. \& Park, N.-G. Morphological and Compositional Progress in Halide Perovskite Solar Cells. Chem Comm 55, 1192-1200 (2019).

4. Ameen, S., Akhtar, M. S., Shin, H.-S. \& Nazeeruddin, M. K. Charge-Transporting Materials for Perovskite Solar Cells. Adv. Inorg. Chem. 72, 185-246 (2018).

5. Fakharuddin, A. et al. Perovskite-Polymer Blends Influencing Microstructures, Nonradiative 
Recombination Pathways, and Photovoltaic Performance of Perovskite Solar Cells. ACS Appl. Mater. Interfaces 10, 42542-42551 (2018).

6. Mingorance, A. et al. Interfacial Engineering of Metal Oxides for Highly Stable Halide Perovskite Solar Cells. Adv. Mater. Interfaces 1800367, 1-10 (2018).

7. You, P., Tang, G. \& Yan, F. Two-dimensional materials in perovskite solar cells. Mater. Today Energy 11, 128-158 (2019).

8. Li, T. et al. Additive engineering for highly efficient organic-inorganic halide perovskite solar cells: Recent advances and perspectives. J. Mater. Chem. A 5, 12602-12652 (2017).

9. Chen, K., Schünemann, S., Song, S. \& Tüysüz, H. Structural effects on optoelectronic properties of halide perovskites. Chem. Soc. Rev. 47, 7045-7077 (2018).

10. Yang, S., Fu, W., Zhang, Z., Chen, H. \& Li, C. Z. Recent advances in perovskite solar cells: Efficiency, stability and lead-free perovskite. J. Mater. Chem. A 5, 11462-11482 (2017).

11. Isabelli, F. et al. Solvent systems for industrial-scale processing of Spiro-OMeTAD hole transport layer in perovskite solar cells. ACS Appl. Energy Mater. 1, 6056-6063 (2018).

12. Wang, Z. K. \& Liao, L. S. Doped Charge-Transporting Layers in Planar Perovskite Solar Cells. Adv. Opt. Mater. 6, 1-13 (2018).

13. Courtier, N. E., Cave, J. M., Foster, J. M., Walker, A. B. \& Richardson, G. How transport layer properties affect perovskite solar cell performance: insights from a coupled charge transport/ion migration model. Energy Environ. Sci. 12, 396-409 (2019).

14. Fakharuddin, A., Schmidt-Mende, L., Garcia-Belmonte, G., Jose, R. \& Mora-Sero, I. Interfaces in perovskite solar cells. Adv. Electron. Mater. 7, 1-44 (2017).

15. Wang, S., Sakurai, T., Wen, W. \& Qi, Y. Energy Level Alignment at Interfaces in Metal Halide Perovskite Solar Cells. Adv. Mater. Interfaces 5, 1-30 (2018).

16. Saidaminov, M. I. et al. Suppression of atomic vacancies via incorporation of isovalent small ions to increase the stability of halide perovskite solar cells in ambient air. Nat. Energy 3, 648-654 (2018).

17. Lee, I., Yun, J. H., Son, H. J. \& Kim, T. S. Accelerated Degradation Due to Weakened Adhesion from LiTFSI Additives in Perovskite Solar Cells. ACS Appl. Mater. Interfaces 9, 7029-7035 (2017).

18. Agresti, A. et al. Graphene Interface Engineering for Perovskite Solar Modules: $12.6 \%$ Power Conversion Efficiency over $50 \mathrm{~cm}^{2}$ Active Area. ACS Energy Lett. 2, 279-287 (2017).

19. Petridis, C., Kakavelakis, G. \& Kymakis, E. Renaissance of graphene-related materials in photovoltaics due to the emergence of metal halide perovskite solar cells. Energy Environ. Sci. 11, 1030-1061 (2018).

20. Taheri, B. et al. Graphene-engineered automated sprayed mesoscopic structure for perovskite device scaling-up. 2D Mater. 5, (2018).

21. Arora, N. et al. Perovskite solar cells with CuSCN hole extraction layers yield stabilized efficiencies greater than 20\%. Science 358, 768-771 (2017).

22. Konios, D. et al. Highly efficient organic photovoltaic devices utilizing work-function tuned graphene oxide derivatives as the anode and cathode charge extraction layers. J. Mater. Chem. A 4, 16121623 (2016).

23. Agresti, A. et al. Efficiency and Stability Enhancement in Perovskite Solar Cells by Inserting LithiumNeutralized Graphene Oxide as Electron Transporting Layer. Adv. Funct. Mater. 26, 2686-2694 (2016). 
24. Agresti, A. et al. Two-dimensional (2D) Material Interface Engineering for Efficient Perovskite Largearea Modules. ACS Energy Lett. 4, 1862-1871 (2019).

25. Najafi, L. et al. $\mathrm{MoS}_{2}$ Quantum Dot/Graphene Hybrids for Advanced Interface Engineering of a $\mathrm{CHNH}_{3} \mathrm{Pbl}_{3}$ Perovskite Solar Cell with an Efficiency of over 20\%. ACS Nano 12, 10736-10754 (2018).

26. Hantanasirisakul, K. \& Gogotsi, Y. Electronic and Optical Properties of 2D Transition Metal Carbides and Nitrides (MXenes). Adv. Mater. 1804779, 1-30 (2018).

27. Naguib, M., Mochalin, V. N., Barsoum, M. W. \& Gogotsi, Y. 25th Anniversary Article : MXenes : A New Family of Two-Dimensional Materials. Adv. Mater. 26, 992-1005 (2013).

28. Zhang, C. J. et al. Transparent, Flexible, and Conductive 2D Titanium Carbide (MXene) Films with High Volumetric Capacitance. Adv. Mater. 29, 1-9 (2017).

29. Lipatov, A. et al. Elastic properties of $2 \mathrm{D} \mathrm{Ti}{ }_{3} \mathrm{C}_{2} \mathrm{~T}_{x} \mathrm{MXene}$ monolayers and bilayers. Sci. Adv. 4, eaat0491 (2018).

30. Akuzum, B. et al. Rheological Characteristics of 2D Titanium Carbide ( MXene ) Dispersions : A Guide for Processing MXenes. ACS Nano 12, 2685-2694 (2018).

31. Khazaei, M., Ranibar, A., Arai, M., Sasaki, T. \& Yunoki, S. Electronic properties and application of MXenes: a theoretical review. J. Mater. Chem. C 5, 2488-2503 (2017).

32. Khazaei, M. et al. OH-terminated two-dimensional transition metal carbides and nitrides as ultralow work function materials. Phys. Rev. B - Condens. Matter Mater. Phys. 92, 1-10 (2015).

33. Hu, T. et al. Chemical Origin of Termination-Functionalized MXenes: $\mathrm{Ti}_{3} \mathrm{C}_{2} \mathrm{~T}_{2}$ as a Case Study. J. Phys. Chem. C 121, 19254-19261 (2017).

34. Liu, Y., Xiao, H. \& Goddard, W. A. Schottky-Barrier-Free Contacts with Two-Dimensional Semiconductors by Surface-Engineered MXenes. J. Am. Chem. Soc. 138, 15853-15856 (2016).

35. Schultz, T. et al. Surface termination dependent work function and electronic properties of $\mathrm{Ti}_{3} \mathrm{C}_{2} \mathrm{~T}_{\mathrm{x}}$ MXene. Chem. Mater. acs.chemmater.9b00414 (2019). doi:10.1021/acs.chemmater.9b00414

36. Yu, Z. et al. MXenes with tunable work functions and their application as electron- and holetransport materials in non-fullerene organic solar cells. J. Mater. Chem. A (2019). doi:10.1039/C9TA01195A

37. Fu, H. C. et al. MXene-Contacted Silicon Solar Cells with $11.5 \%$ Efficiency. Adv. Energy Mater. 1900180, 1-9 (2019).

38. Dall'Agnese, C., Dall'Agnese, Y., Anasori, B., Sugimoto, W. \& Mori, S. Oxidized $\mathrm{Ti}_{3} \mathrm{C}_{2} \mathrm{MXene}$ nanosheets for dye-sensitized solar cells. New J. Chem. 42, 16446-16450 (2018).

39. Guo, Z. et al. High Electrical Conductivity 2D MXene Serves as Additive of Perovskite for Efficient Solar Cells. Small 1802738, 1-8 (2018).

40. Yang, L. et al. $\mathrm{SnO}_{2}-\mathrm{Ti}_{3} \mathrm{C}_{2} \mathrm{MXene}$ electron transport layers for perovskite solar cells. J. Mater. Chem. A (2019). doi:10.1039/C8TA12140K

41. Peng, C. et al. High efficiency photocatalytic hydrogen production over ternary $\mathrm{Cu} / \mathrm{TiO}_{2} @ \mathrm{Ti}_{3} \mathrm{C}_{2} \mathrm{~T}_{2}$ enabled by low-work-function 2D titanium carbide. Nano Energy 53, 97-107 (2018).

42. Deng, W. et al. All-Sprayed-Processable, Large-Area, and Flexible Perovskite/MXene-Based Photodetector Arrays for Photocommunication. Adv. Opt. Mater. 1801521, 1-9 (2019).

43. Philippe, B. et al. Valence Level Character in a Mixed Perovskite Material and Determination of the Valence Band Maximum from Photoelectron Spectroscopy: Variation with Photon Energy. J. Phys. 
Chem. C 121, 26655-26666 (2017).

44. Ahn, N. et al. Trapped charge-driven degradation of perovskite solar cells. Nat. Commun. 7, 1-9 (2016).

45. Auf Der Maur, M. et al. The multiscale paradigm in electronic device simulation. IEEE Trans. Electron Devices 58, 1425-1432 (2011).

46. Deepa, M. et al. Cesium power: Low $\mathrm{Cs}^{+}$levels impart stability to perovskite solar cells. Phys. Chem. Chem. Phys. 19, 4069-4077 (2017).

47. Sarycheva, A. et al. Two-Dimensional Titanium Carbide (MXene) as Surface-Enhanced Raman Scattering Substrate. J. Phys. Chem. C 121, 19983-19988 (2017).

48. Chaudhuri, K. et al. Highly Broadband Absorber Using Plasmonic Titanium Carbide (MXene). ACS Photonics 5, 1115-1122 (2018).

49. Alhabeb, M. et al. Guidelines for Synthesis and Processing of Two-Dimensional Titanium Carbide $\left(\mathrm{Ti}_{3} \mathrm{C}_{2} \mathrm{~T}_{x} \mathrm{MXene}\right)$. Chem. Mater. 29, 7633-7644 (2017).

\section{METHODS}

\section{MAX phase and MXene preparation}

$\mathrm{Ti}_{3} \mathrm{AlC}_{2} \mathrm{MAX}$ phase was synthesized as described elsewhere..$^{49}$ In short, commercial powders of $\mathrm{Ti}, \mathrm{Al}$, and TiC were mixed together to achieve 3.0:1.1:1.9 molar ratio of Ti:Al: $\mathrm{C}$ in glass jar for $24 \mathrm{~h}$ using zirconia balls. The mixture was put in corundum crucible and heated to $1400{ }^{\circ} \mathrm{C}$ for $2 \mathrm{~h}$ under Ar atmosphere in tube furnace (Carbolite). The obtained MAX compact was crashed and sieved through a 400 mesh size sieve (stainless steel, Fritsch) to get a powder with an average particle size less than $30 \mu \mathrm{m} . \mathrm{Ti}_{3} \mathrm{C}_{2} \mathrm{~T}_{\mathrm{x}} \mathrm{MXene}$ was synthesized by A-layer selective etching from the $\mathrm{Ti}_{3} \mathrm{AlC}_{2}$ precursor via less aggressive method, known as the minimally intensive layer delamination (MILD). ${ }^{49}$ We used $1 \mathrm{~g}$ of fine-ground MAX phase powder and slowly added it in a plastic jar with as-prepared mixture of $1 \mathrm{~g} \mathrm{LiF}$ and $20 \mathrm{ml}$ of $6 \mathrm{M} \mathrm{HCL}$. The etching lasted for $24 \mathrm{~h}$ at $30-35^{\circ} \mathrm{C}$, while being stirred by a magnetic stir bar. After that, the etched powder was repeatedly washed with deionized water and centrifuged until neutral $\mathrm{pH}(\sim 6-7)$ was reached. The $\mathrm{Ti}_{3} \mathrm{C}_{2} \mathrm{~T}_{\mathrm{x}}$ colloidal suspension in acetonitrile (ACN) was obtained by washing and centrifuging MXenes in ACN for several times to remove water. The MXene suspension was ice-cooled bath sonicated at $35 \mathrm{kHz}$ for $1 \mathrm{~h}$ (Laborette 17, Fritsch) under continuous argon bubbling followed by $1 \mathrm{~h}$ of centrifugation at $3500 \mathrm{rpm}$ to extract single-layer flakes.

\section{MXene characterization}

A transmission electron microscope, TEM, (JEOL JEM-2100, Japan) with an accelerating voltage of $200 \mathrm{kV}$ was used to characterize structure of $\mathrm{Ti}_{3} \mathrm{C}_{2} \mathrm{~T}_{x}$. TEM samples were prepared by dropping delaminated $\mathrm{Ti}_{3} \mathrm{C}_{2} \mathrm{~T}_{\mathrm{x}}$ suspension onto a copper grid and dried in the air. The chemical composition of the MXenesurface was characterized by X-ray photoelectron spectroscopy (XPS) using an Axis Supra (Kratos Analytical, UK) spectrometer. The peak fitting was performed using a Gaussian-Lorentzian peak shape after the subtraction of a Shirley background by the CasaXPS software (version 2.3.17). X-Ray diffraction (XRD) spectra of MXene and $\mathrm{TiO}_{2}$ samples were collected using a Rigaku Miniflex $600 \mathrm{X}$-ray diffractometer with monochromatic $\mathrm{Cu}$ $K_{\alpha}$ radiation $(\lambda=1.5406 \AA)$.

\section{Device realization}

Materials 
Mesoporous transparent titania paste (30 NR-D), formamidinium iodide (FAl) and methylammonium bromide (MABr) are purchased from GreatCell Solar, while Lead(II) iodide ( $\left.\mathrm{Pbl}_{2}\right)$, Lead(II) Bromide $\left(\mathrm{PbBr}_{2}\right)$, and cesium iodide (CsI) complex are ordered from TCl and GmbH respectively. Moreover Cobalt(III) FK209 is purchased by Lumtec, while 2,20,7,70-tetrakis-(N,N-dip-methoxyphenylamine)9,9'-spirobifluorene (SpiroMeOTAD) is from Borun. All other materials including titanium(IV) isopropoxide (TTIP), lithium bis(trifluoromethanesulfonyl)imide (Li-TFSI), acetylacetone, ethanol, acetone, dimethylformamide (DMF), dimethyl sulfoxide (DMSO), acetonitrile ( $A C N)$, tert-butylpyridine (tBP), chlorobenzene (CB), are purchased from Sigma-Aldrich. All materials are used as received unless specified otherwise.

\section{Cell fabrication}

Patterned Florine-doped tin oxide (FTO, Pilkington, $8 \Omega \square^{-1}$ ) coated glasses were firstly washed with a cleaning liquid, dissolved in deionized water and then cleaned by ultrasonic bath with acetone and ethanol for 10 minute each step. The compact $\mathrm{TiO}_{2}\left(\mathrm{cTiO}_{2}\right)$ blocking layer $(40 \mathrm{~nm})$ was deposited by spray pyrolysis at $460{ }^{\circ} \mathrm{C}$ and annealed for 30 minutes the same temperature. The $\mathrm{CTiO}_{2}$ solution was composed by acetylacetone ( 2 $\mathrm{mL})$, TTIP $(3 \mathrm{~mL})$ and ethanol $(45 \mathrm{~mL})$ and doped by $\mathrm{Ti}_{3} \mathrm{C}_{2} \mathrm{~T}_{\times} \mathrm{MXene}$ dispersion $(0.07 \mathrm{mg} / \mathrm{ml})$ insertion into the standard $\mathrm{CTiO}_{2}$ solution with a volume ratio of $1 \%$. ( $\left.\mathrm{cTiO}_{2}+\mathrm{MXene}\right)$ The same MXene suspension, with the $0.42 \%$ volume ratio was used to dope the solution of mesoporous $\mathrm{TiO}_{2}\left(\mathrm{mTiO}_{2}+\mathrm{MXene}\right)$ paste $30 \mathrm{NR}-\mathrm{D}$ in ethanol (1:10 weight ratio). This $\mathrm{MXene-doped} \mathrm{mesoporous} \mathrm{TiO}_{2}\left(\mathrm{mTiO}_{2}+\mathrm{MXene}\right)$ solution was deposited on the substrate by spin-coating at $3000 \mathrm{rpm}$ for $20 \mathrm{~s}$ and subsequently annealed in air for $30 \mathrm{~min}$ at $480^{\circ} \mathrm{C}$ by obtaining a $120 \mathrm{~nm}$ thick scaffold layer. The MXene interlayer was realized by spraying the MXene suspension $\left(0.03 \mathrm{ml} / \mathrm{cm}^{2}\right)$ by an airbrush onto the $80^{\circ} \mathrm{C}$ pre-heated substrate. The sample was immediately transferred to a $\mathrm{N}_{2}$-filled glove box where was deposited the perovskite layer. The precursor solution, composed by a mix of FAl (1 M), $\mathrm{Pbl}_{2}(1.1 \mathrm{M}), \mathrm{MABr}(0.2 \mathrm{M}), \mathrm{PbBr}_{2}(0.2 \mathrm{M})$ and $\mathrm{Csl}(1.5 \mathrm{M}$ stock solution in DMSO) in the mixture of anhydrous DMF/DMSO (4:1 vol/vol) was stirred at room temperature for $30 \mathrm{~min}$, and then was doped by adding $0.014 \mathrm{mg}$ of MXene in $1 \mathrm{ml}$ perovskite precursor solution. The MXene doping amount has been optimized in term of cell PCE, as reported in Figure S25. The MXene-doped perovskite solution was spin coated onto the samples with a one-step deposition and anti-solvent method, consisting in a two steps program at 1000 and $5000 \mathrm{rpm}$ for $10 \mathrm{~s}$ and $30 \mathrm{~s}$ respectively. During the second step, $200 \mu \mathrm{L}$ of $\mathrm{CB}$ was poured on the spinning substrate $7 \mathrm{~s}$ prior to the end of the program. Immediately after spin coating, the substrates are annealed at $100^{\circ} \mathrm{C}$ for $1 \mathrm{~h}$ to form a perovskite crystal structure and by obtaining a compact perovskite layer 450nm thick. After that, a spiro-OMeTAD $\left(73.5 \mathrm{~g} \cdot \mathrm{L}^{-1}\right)$ in CB solution doped with tBP (26.7 $\left.\left.\mu \mathrm{L} \cdot \mathrm{mL}^{-1}\right)\right)$, LiTFSI $\left(16.6 \mu \mathrm{L} \cdot \mathrm{mL}^{-1}\right)$ and a Cobalt(III) FK209 complex $\left(7.2 \mu \mathrm{L} \cdot \mathrm{mL}^{-1}\right)$ was spin-coated at $2000 \mathrm{rpm}$ for $20 \mathrm{~s}$ by achieving a hole transport layer of about $140 \mathrm{~nm}$. The precursor solution concentrations of LiTFSI are $520 \mathrm{mg} / \mathrm{ml}$ in ACN and $375.8 \mathrm{mg} / \mathrm{ml}$ in ACN for Cobalt (III) FK209 respectively. Finally, the device was completed by the high-vacuum thermal evaporation of a gold counter electrode ( $100 \mathrm{~nm})$ on an active area of $0.09 \mathrm{~cm}^{2}$ defined by a shadow mask.

\section{Pristine and MXene-doped perovskite characterization}

The UPS and XPS measurements were carried out at the Material Science Laboratory of the LNF-INFN (Frascati, Italy) on pristine and MXene-doped perovskite films deposited on a $\mathrm{TiO}_{2} / \mathrm{FTO}$ substrate by exciting the sample with the Hell $\alpha(40.81 \mathrm{eV})$ and $\mathrm{Mg} \mathrm{K} \alpha(1253.6 \mathrm{eV})$ radiation, respectively, and collecting the photoelectrons with a Omicron EA 125 hemispherical analyzer. For the WF measurements a bias of $8 \mathrm{~V}$ was applied to the sample. All spectra were calibrated versus the Fermi level at zero binding energy measured on a clean metal surface. 
Scanning Electron Microscopy: Electrodes were imaged by aim of a field-emission SEM (JOEL JSM-7500 FA). The acceleration voltage was set at $5 \mathrm{kV}$. Images were collected using the in-lens sensors (secondary electron in-lens image) and the secondary electron sensor (lower secondary electron image). No coating was applied.

\section{Device characterization}

Electro-Optical measurements: IPCE, ARKEO, I-V characteristics.

Current-Voltage (I-V) characteristics of masked and encapsulated devices are acquired in air atmosphere by using a solar simulator (ABET Sun 2000, class A) at AM1.5 and $100 \mathrm{~mW} \mathrm{~cm}^{-2}$ illumination conditions, calibrated with a certified reference Si Cell (RERA Solutions RR-1002). Incident power was measured with a Skye SKS 1110 sensor. The class was measured with a BLACK-Comet UV-vis spectrometer. The devices were not preconditioned before the J-V measurements and a mask of $0.09 \mathrm{~cm}^{2}$ was placed atop the solar cells; any variation of short circuit current density was observed in the case of smaller measurement masks. Both reverse and forward I-V scans were performed by using a scan rate of $20 \mathrm{mV} / \mathrm{s}$ and a dwell time of $200 \mathrm{~ms}$. Any spectral mismatch factor correction was applied to PCE measurements since the class A Abet Sun Simulator employed for the I-V characterization has an error $<1.3 \%$ in the spectral region of interest between 400 and $900 \mathrm{~nm}$, that does not affect significantly the performance measurements accuracy.

Illumination intensity dependence of $\mathrm{V}_{\mathrm{OC}}$ and dark JV measurements are performed with a modular testing platform (Arkeo - Cicci research s.r.I.) composed by a white LED array (4200Kelvin) tuneable up to $200 \mathrm{mWcm}^{-}$ ${ }^{2}$ of optical power density and a high speed source meter unit (600 Ksamples/s) in a four wire configuration. A spring contact based sample holder is used to improve the repeatability of the experiments. Transient measurements are performed in a high perturbation configuration by acquiring the entire $V_{o c}$ rise profile after switching the light intensity from $\sim 0$ to 1 Sun.

IPCE spectra acquisition are carried out by means of a homemade setup composed by a monochromator (Newport, mod. 74000) coupled with a xenon lamp (Oriel Apex, Newport) and a source meter (Keithley, mod. 2612). A home-made LabVIEW program controls the spectra acquisition.

\section{DFT calculations and device simulations}

First-principles calculations based on density functional theory (DFT) within the local density approximation (LDA) are performed using the Quantum Espresso package. Scalar-relativistic norm-conserving pseudopotentials are employed, with the exchange-correlation energy parameterized by Perdew-Zunger. Device simulations are performed by using TiberCad multiscale simulation software (TiberLAB s.r.l.).

DATA AND CODE AVAILABILITY. The experimental data, Quantum Espresso scripts for DFT calculation and TiberCad scripts for device simulations that support the findings of this study are available from the corresponding authors upon reasonable request.

\section{REFERENCES}

50. Alhabeb, M. et al. Guidelines for Synthesis and Processing of Two-Dimensional Titanium Carbide $\left(\mathrm{Ti}_{3} \mathrm{C}_{2} \mathrm{~T}_{x}\right.$ MXene). Chem. Mater. 29, 7633-7644 (2017). 